\title{
Analisi di costo efficacia nella terapia della BPCO
}

Sergio Iannazzo§, Lorenzo Pradelli§, Roberto W. Dal Negro\#, Mario Eandi*

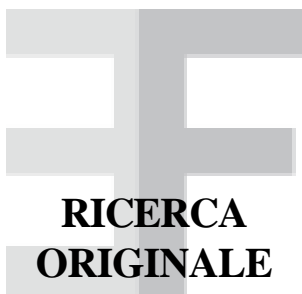

\begin{abstract}
Current practice guidelines for the treatment of COPD recommend the use of combined inhaled corticosteroids and long-acting bronchodilators in severe and very severe patients (GOLD stages III and IV).

OBJECTIVES: To analyze the economical and clinical impact of this recommendation, the affordability of its widespread application, as well as the relative pharmacoeconomical performance of the available options for severe and very severe COPD in Italy.

METHODS: Published data on the Italian COPD population were fitted in a disease progression model based on a Markov chain representing severity stages and death. Alternative therapeutic options (salmeterol/ fluticasone - SF, formoterol/budesonide - FB, salmeterol alone - S, fluticasone alone - F and control - C) were represented as competing arms in a decision tree. Efficacy data from international trials were expressed in terms of risk reduction. Clinical parameters used were number of exacerbations and symptom-free days. Direct and indirect costs were considered and valued according to present prices and tarifs. The analyses were conducted from National Health System, societal and patient perspectives with time horizons of 1,5, and 10 years, and lifelong.

RESULTS: The yearly total direct costs of treating COPD patients in Italy is estimated in approximately 7 billion Euro, with a mean cost/patient/year around 2,400 Euro. Mean survival of the cohort is 11,5 years. The $\mathrm{C}$ and $\mathrm{F}$ strategies are dominated (i.e. are associated with worse outcomes and higher costs) by all alternatives. S/F and F/B are the most effective strategies, with a slight clinical superiority of the latter, but are also marginally more expensive than $\mathrm{S}$. Incremental cost/effectiveness of S/F vs. S is 679,55 Euro/avoided exacerbation and 3,31Euro/gained symptom-free day.

CONCLUSIONS: The recommended use of combined inhaled corticosteroids and long-acting bronchodilators for severe and very severe COPD patients, as compared with current practice, has the potential of improving clinical outcomes without increasing health care costs.
\end{abstract}

Keywords: inhaled corticosteroids, long-acting bronchodilators, Chronic Obstructive Pulmonary Disease (COPD)

Farmeconomia e percorsi terapeutici 2005; 6 (4): 277-287

\section{INTRODUZIONE}

La broncopneumopatia cronica ostruttiva (BPCO) è la terza causa di mortalità nei paesi industrializzati, Italia compresa, e tra le prime cause di invalidità. L'impatto socio-economico di questa malattia è pesantissimo, come evidente anche dal fatto che nel nostro Paese rappresenta la settima causa di ricovero ospedaliero (quarta tra le malattie mediche) ed è al quarto posto per numero di giornate di degenza. La principale causa di ricovero nei pazienti con BPCO è la "riacutizzazione", evento clinico a definizione puramente sintomatica, che comprende uno spettro piuttosto ampio di manifestazioni, dal semplice aumento della secrezione mucosa e della tosse a forme di dispnea gravemente invalidanti e potenzial- mente fatali. L'unico intervento che si è dimostrato efficace nel modificare il decorso progressivo della malattia è la sospensione del fumo di sigaretta, mentre gli interventi farmacologici hanno lo scopo di migliorare la qualità di vita del paziente, mediante il controllo o la riduzione della sintomatologia e la riduzione della frequenza di riacutizzazione.

Le linee guida GOLD (Global Initiative on Chronic Obstructive Lung Disease), elaborate dal National Institute of Health statunitense e dall'OMS, tramite il coinvolgimento di un panel internazionale di esperti, raccomandano l'utilizzo di corticosteroidi per via inalatoria in associazione ai broncodilatatori a lunga durata d'azione nei pazienti con BPCO grave e molto grave, sulla base delle evidenze che ne indica-
* Farmacologia Clinica, Università di Torino

\# ASL 22 Ospedale Orlandi, Bussolengo, Verona

$\S$ Advanced Research, Torino 


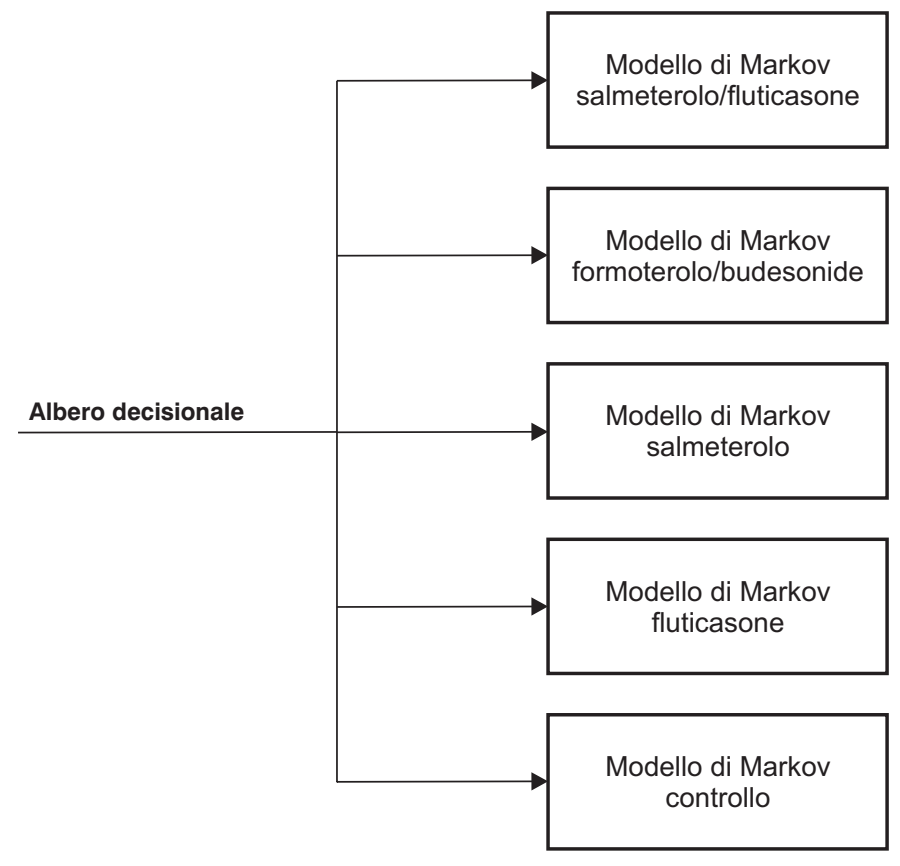

Figura 1

Struttura dell'albero decisionale no l'efficacia in termini di miglioramento della qualità di vita e riduzione del numero delle riacutizzazioni.

Le conseguenze economiche dell'adozione universale di questa strategia nella popolazione di broncopneumopatici cronici italiani non sono note.

\section{OBIETTIVI}

Lo scopo del presente lavoro è di valutare le conseguenze cliniche ed economiche dell'aderenza alla strategia raccomandata dalle linee guida GOLD per i pazienti con BPCO grave e molto grave (stadi III e IV, FEV $1<50 \%$ del valore teorico previsto) nel contesto sanitario italiano.

\section{MATERIALIEMETODI}

Abbiamo progettato e costruito un modello farmacoeconomico analitico a struttura mista, in cui il problema della scelta tra le alternative terapeutiche disponibili è rappresentato da un albero decisionale, i cui rami sono costituiti da identiche catene di Markov che simulano la progressione della malattia su un medesimo gruppo di individui.

\section{Opzioni terapeutiche}

Le alternative terapeutiche prese in considerazione sono state:

- nessuna variazione della terapia attuale (controllo-C);

- strategia GOLD, con aggiunta di un'associazione inalatoria precostituita di betaagonista a lunga durata d'azione e corticosteroide (salmeterolo/fluticasone 50/ $500 \mu \mathrm{g}$ bid - SF e formoterolo/budesonide 2 x 160/4,5 $\mu \mathrm{g}$ bid-FB);

- aggiunta del solo corticosteroide inalatorio alla terapia attuale (fluticasone $500 \mu \mathrm{g}$ bid $-\mathrm{F}$ );

- utilizzo del solo beta-agonista a lunga durata d'azione (salmeterolo $50 \mu \mathrm{g}$ bid $-\mathrm{S}$ ).

L'evoluzione clinica di ogni gruppo di pazienti esposti alle varie alternativi decisionale

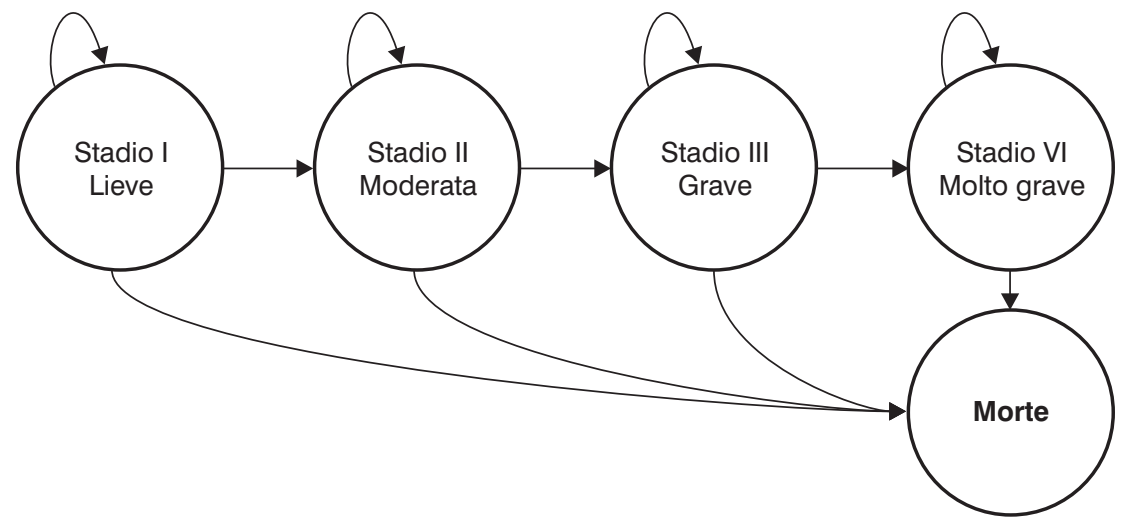




\begin{tabular}{ccccc}
\hline Fascia d'età & Popolazione & $\begin{array}{c}\text { Prevalenza } \\
\text { BPCo }\end{array}$ & $\begin{array}{c}\text { Popolazione } \\
\text { con BPCO }\end{array}$ & Fonte \\
\hline $20-45$ & 21.293 .000 & $1,30 \%$ & 276.809 & De Marco et al. [1] \\
$46-55$ & 7.579 .000 & $6 \%$ & 454.740 & Viegi et al. [2] \\
$56-65$ & 6.737 .000 & $11 \%$ & 741.070 & Viegi et al. [2] \\
$65+$ & 10.097 .000 & $14,10 \%$ & 1.423 .677 & ISTAT [3] \\
Totale & & & 2.896 .296 & \\
\hline
\end{tabular}

è modellizata mediante una struttura di Markov, che rappresenta la dinamica delle transizioni tra gli stadi della malattia e la morte. Le cinque catene di Markov hanno le stesse caratteristiche per quel che riguarda gli stati, la distribuzione iniziale di popolazione e le probabilità di transizione tra stati. Differiscono, invece, per quel che riguarda i costi e i guadagni, che sono funzione del trattamento farmacologico, comunque applicato solo negli stadi III e IV. La durata del singolo ciclo è di un anno.

I costi e i guadagni sono considerati sempre come grandezze di transizione, per cui vengono attribuiti nel passaggio da uno stato all'altro (considerando come passaggio anche la permanenza nello stesso stato); la simulazione del modello continua fino all'avvenuta transizione di tutti i soggetti allo stato di morte.

\section{Popolazione iniziale}

Le caratteristiche del gruppo di individui che popola gli stati del modello all'inizio del primo ciclo (popolazione iniziale) sono state costruite in modo da replicare il più fedelmente possibile l'insieme dei malati di BPCO in Italia, sulla base della letteratura disponibile.

Da uno studio internazionale [1] su adulti di età medio giovane si rileva una prevalenza in Italia di 1,3\% nella fascia di età compresa tra 20 e 44 anni. Un altro studio [2], effettuato nel nord Italia, riporta una prevalenza del $6 \%$ nella fascia d'età compresa tra 46 e 55 anni e una di $11 \%$ per la fascia tra 56 e 65. Infine una indagine multiscopo ISTAT [3] ha rilevato una prevalenza del $14,1 \%$ per la fascia di popolazione anziana oltre i 65 anni. Applicando questi dati alla popolazione generale italiana (dati ISTAT 1999-2000) si arriva ad una stima complessiva di circa 2.900.000 individui con BPCO.

In assenza di dati sulla stadiazione in classi GOLD 2004 della popolazione italiana con $\mathrm{BPCO}$, la distribuzione per classi di gravità ha riprodotto quella riportata per pazienti inglesi e statunitensi al Congresso della European Respiratory Society del 2004 [4] (stadio I 42\%, stadio II $41 \%$, stadio III $13 \%$ e stadio IV $4 \%$ del totale dei broncopneumopatici cronici).

Il valore medio di FEV1 per ogni stadio è stato ricavato da dati dello studio Salute Respiratoria nell'Anziano (SaRA) [5]. Infine il numero medio di riacutizzazioni /paziente/anno per stadio è stato ricavato da una rielaborazione di dati dello studio Inhaled Steroids in Obstructive Lung Disease in Europe (ISOLDE) [6].

\section{Probabilità di transizione}

Per ogni paziente incluso nel modello, la probabilità di passare allo stato successivo è calcolata mediante un modello di progressione della malattia che stima il numero di anni necessario per passare, in media, allo stadio successivo: partendo dalla riduzione della capacità polmonare di un malato di BPCO, assunto come pari al valore medio per un fuma-

\section{Tabella I}

Composizione delle stime di prevalenza della BPCO nella popolazione italiana

\begin{tabular}{|c|c|c|c|c|c|c|}
\hline & Stadio I & Stadio II & Stadio III & Stadio IV & Totale & Fonte \\
\hline$\%$ pop & $42 \%$ & $41 \%$ & $13 \%$ & $4 \%$ & $100 \%$ & ERS [4] \\
\hline $\begin{array}{l}\text { Popolazione } \\
\text { iniziale }\end{array}$ & 1.216 .444 & 1.187 .481 & 376.518 & 115.852 & 2.896 .296 & vd. tab. I \\
\hline $\begin{array}{l}\text { FEV1 medio } \\
\text { (\% pred.) }\end{array}$ & $89,80 \%$ & $63,70 \%$ & $40,00 \%$ & $26,20 \%$ & & SaRA [5] \\
\hline $\mathrm{N}$ riac/paz/anno & 0 & 0,8 & 1,8 & 2,6 & & ISOLDE [6] \\
\hline
\end{tabular}

Tabella II

Composizione e caratteristiche della popolazione iniziale per il modello 


\begin{tabular}{|c|c|c|c|c|}
\hline & Stadio I & Stadio II & Stadio III & Stadio IV \\
\hline \multicolumn{5}{|c|}{ Probabilità annuale di passare allo stato successivo } \\
\hline & $7,7 \%$ & $7,1 \%$ & $11,1 \%$ & $0,0 \%$ \\
\hline \multicolumn{5}{|c|}{ Probabilità annuale di morte } \\
\hline anno 1 & $1,13 \%$ & $2,42 \%$ & $6,42 \%$ & $10,05 \%$ \\
\hline anno 2 & $1,25 \%$ & $2,69 \%$ & $7,13 \%$ & $11,15 \%$ \\
\hline anno 3 & $1,40 \%$ & $2,99 \%$ & $7,93 \%$ & $12,40 \%$ \\
\hline anno 4 & $1,55 \%$ & $3,33 \%$ & $8,82 \%$ & $13,80 \%$ \\
\hline anno 5 & $1,74 \%$ & $3,73 \%$ & $9,88 \%$ & $15,46 \%$ \\
\hline anno 6 & $1,93 \%$ & $4,13 \%$ & $10,96 \%$ & $17,15 \%$ \\
\hline anno 7 & $2,12 \%$ & $4,54 \%$ & $12,02 \%$ & $18,81 \%$ \\
\hline anno 8 & $2,35 \%$ & $5,03 \%$ & $13,33 \%$ & $20,85 \%$ \\
\hline anno 9 & $2,58 \%$ & $5,53 \%$ & $14,62 \%$ & $22,87 \%$ \\
\hline anno 10 & $2,87 \%$ & $6,15 \%$ & $16,24 \%$ & $25,41 \%$ \\
\hline anno 11 & $3,21 \%$ & $6,87 \%$ & $18,13 \%$ & $28,37 \%$ \\
\hline anno 12 & $3,60 \%$ & $7,70 \%$ & $20,32 \%$ & $31,80 \%$ \\
\hline anno 13 & $3,96 \%$ & $8,47 \%$ & $22,31 \%$ & $34,92 \%$ \\
\hline anno 14 & $4,43 \%$ & $9,47 \%$ & $24,87 \%$ & $38,94 \%$ \\
\hline anno 15 & $4,89 \%$ & $10,44 \%$ & $27,35 \%$ & $42,84 \%$ \\
\hline
\end{tabular}

Tabella III

Principali probabilità di transizione utilizzate nel modello

tore (parere esperto), cioè a circa $45 \mathrm{ml} /$ anno [7], si è ricavato il progressivo declino del FEV1, espresso come percentuale del valore teorico (calcolato mediante l'equazione di Crapo et al. $[10,11,12]$ a partire da altezza ed età media). L'inverso del numero medio di anni in cui un soggetto di un determinato stadio passa allo stadio successivo è la probabilità di passare allo stato successivo del modello.

La mortalità (o, meglio, probabilità di passare allo stato morte) è variabile ad ogni ciclo ed è calcolata applicando un rischio relativo (costante per ogni stadio) ai tassi di mortalità ISTAT della popolazione italiana [8].

Il rischio relativo è stato ricavato a partire dal dato di sopravvivenza a 4 anni di una coorte di malati di BPCO classificati secondo le linee guida ATS (gli stadi corrispondono approssimativamente a quelli GOLD [9]). Per lo stadio I il coefficiente è stato assunto uguale a 1 (nessun incremento della mortalità naturale a causa della malattia). La probabilità di rimanere nello stesso stato, infine, è il complemento a 1 della somma delle altre due.

\section{Efficacia e guadagni}

L'efficacia dei trattamenti farmaceutici valutati è derivata da due RCT internazionali, uno relativo all'associazione SF (Calverley et al.) [13] e uno relativo all'associazione FB (Szafranski et al.) [14]. Un altro studio pilota, condotto in Italia (Dal Negro et al.) [15] su SF, ha presentato risultati sostanzialmente sovrapponibili agli studi già citati.

Un ulteriore studio internazionale [16], che avrebbe potuto essere utilizzato per l'associazione FB (con risultati di efficacia praticamente equivalenti), è stato scartato per la presenza di una fase di run-in per l'ottimizzazione del trattamento prima dell'inizio del trial, fase assente negli altri due studi.

Nel complesso, questi studi confermano il parere generalmente espresso ed esplicitato nelle linee guida GOLD, secondo cui nessun trattamento farmacologico oggi disponibile è in grado di modificare sostanzialmente il decorso progressivo della malattia. L'obiettivo primario della terapia farmacologica della BPCO è infatti il controllo della sintomatologia e la riduzione delle esacerbazioni. Di conseguen$\mathrm{za}$, come indicatori di efficacia del trattamento si sono considerati:

- il numero medio di riacutizzazioni/anno;

- il numero medio di giorni liberi da sintomi, misurato come il numero di giorni in cui il paziente non fa uso di "relief medications".

In Tabella IV e V sono riportati, per i due studi, i dati dei pazienti all'inizio del trial e il numero medio di riacutizzazioni alla fine del trial nei diversi gruppi di trattamento.

Rispetto allo studio Szafranski, lo studio Calverley ha arruolato anche pazienti con BPCO in stadi meno avanzati, per cui i trattamenti studiati non riportano l'indicazione in scheda tecnica e non sono raccomandati dalle linee guida cui abbiamo fatto riferimento. Abbiamo quindi utilizzato i dati dei pazienti con un FEV1 basale $<50 \%$, che corrisponde al criterio di inclusione nello studio Szafranski e alla gravità di malattia in cui iniziano a essere somministrate le associazioni di corticosteroidi e broncodilatatori nel nostro modello. Per il salmeterolo e il fluticasone utilizzati in monoterapia il dato relativo al sottogruppo di pazienti più gravi non è disponibile, per cui si è considerato il dato complessivo. Ne risulta un rischio relativo di riacutizzazione, rispetto al gruppo controllo, di 0,7, 0,758, 0,8 e 0,81 per $\mathrm{SF}, \mathrm{FB}, \mathrm{S}$ e F, rispettivamente. Anche il numero medio di giorni all' anno liberi da sintomi è sta- 
to ottenuto dagli studi Calverley et al. e Szafranski et al., mentre la riduzione dell'uso giornaliero di "relief medications", che è di circa il $50 \%$ per SF in Calverley et al, non è presente nello studio Szafranki, per cui l'efficacia di FB nel ridurre il ricorso ai broncodilatatori a breve durata d'azione è stato assunto pari a $\mathrm{SF}$, cioè del $50 \%$.

I dati di efficacia, per essere utilizzati nel modello di Markov, sono stati trasformati in guadagni legati alla permanenza di un soggetto in uno stato:

- numero di riacutizzazioni $\rightarrow$ nel ramo di controllo vengono applicate le probabilità di riacutizzazione/paziente/anno per stato definiti per la popolazione iniziale, mentre nei rami attivi il numero di riacutizzazioni annue è abbattuto moltiplicando il valore del ramo di controllo per i relativi RR;

- numero dei giorni liberi da sintomi $\rightarrow$ nei rami attivi vengono applicate le percentuali di giorni all' anno liberi da sintomi indicate sopra $(3 \%$ S, $2 \%$ F, $14 \%$ SF, $12 \%$ FB). Per il ramo di controllo viene applicata la percentuale dello $0 \%$. Questa assunzione non è sicuramente realistica, soprattutto per gli stadi iniziali della malattia, ma in letteratura manca un dato maggiormente affidabile. Per questa ragione il numero di giorni liberi da sintomi nel ramo di controllo non può essere letto come un dato assoluto e i risultati dei rami attivi del modello devono essere interpretati come dati incrementali.

Si assume che la transizione tra due stati avvenga mediamente a metà ciclo. Pertanto nel passaggio da uno stato all'altro viene applicato un guadagno medio tra $\mathrm{i}$ due caratteristici dei due stati tra cui avviene la transizione.

\section{Costi: prospettive e guadagni}

Gli indicatori di costo da applicare al modello di Markov sono stati elaborati a partire dei dati presentati dalla fase prospettica dello studio ICE (Italian Costs for Exacerbations in COPD) [17].

In particolare, lo studio ICE ha rilevato una serie di costi legati alle riacutizzazioni e una serie di costi non legati alle riacutizzazioni, entrambi suddivisi in costi diretti e indiretti.

La nostra rielaborazione è iniziata con un ricalcolo dei costi per esami diagnostici e terapie riabilitative, che sono stati aggiornati sostituendo le tariffe ministeriali nazionali (non più aggiornate dal 1996 e non utilizzate da gran parte delle regioni) con quelle vigenti in Piemonte dal 2004

Abbiamo inoltre riconsiderato la voce di costo dei farmaci respiratori non associata a riacutizzazioni, ossia legato al trattamento di base della malattia. Tale voce $(419,80$ euro/

\begin{tabular}{|c|c|c|c|c|}
\hline $\begin{array}{l}\text { Salmeterolo- } \\
\text { fluticasone }\end{array}$ & Placebo & Salmeterolo & Fluticasone & SF \\
\hline Arruolati & 361 & 372 & 374 & 358 \\
\hline Ritirati & 140 & 119 & 108 & 89 \\
\hline Età media & 63,4 & 63,2 & 63,5 & 62,7 \\
\hline $\begin{array}{l}\text { Postbronchodilator } \\
\text { FEV1 (mL) }\end{array}$ & 1.379 & 1.346 & 1.363 & 1.419 \\
\hline $\begin{array}{l}\text { N. medio } \\
\text { riac/paziente/anno }\end{array}$ & 1,3 & 1,04 & 1,05 & 0,97 \\
\hline
\end{tabular}

Tabella IV

Dati salienti utilizzati, studio Calverley et al [13]

\begin{tabular}{lcccc}
\hline $\begin{array}{l}\text { Formoterolo- } \\
\text { budesonide }\end{array}$ & Placebo & Formoterolo & Budesonide & FB \\
\hline Arruolati & 205 & 201 & 198 & 208 \\
Ritirati & 90 & 64 & 62 & 59 \\
Età media & 65 & 63 & 64 & 64 \\
$\begin{array}{l}\text { FEV1 (mL) } \\
\text { FEV1 (\% pred) }\end{array}$ & 980 & 1.000 & 1.010 & 960 \\
$\begin{array}{l}\mathrm{N} . \text { medio } \\
\text { riac/paziente/anno }\end{array}$ & 1,87 & 36 & 37 & 36 \\
\hline
\end{tabular}

\section{Tabella V}

Dati salienti utilizzati, studio Szafranski et al [14]

paziente/anno), derivante da un mix di trattamenti non esplicitato nello studio, è stata sostituita dal costo di un trattamento con teofillina e $\beta 2$-agonisti a breve durata. I costi unitari di tale trattamento sono stati derivati dallo studio "The burden of COPD in Italy"[18].

Lo studio ICE classifica i pazienti secondo gli stadi GOLD e mette a disposizione i valori medi - limitatamente ai costi diretti totali - dei singoli stati.

Estrapolando le proporzioni tra costi legati e non legati alle riacutizzazioni e tra costi diretti e indiretti, abbiamo ricostruito il dettaglio dei costi per lo stadio II, III e IV della malattia. Infine abbiamo diviso i costi legati alle riacutizzazioni per il numero medio di riacutizzazioni sperimentato dai pazienti dello studio ICE, per ottenere un costo annuale diretto, indiretto e totale per riacutizzazione, in ogni stadio della malattia. Lo stadio I della malattia non è rappresentato nello studio ICE (assenza di riacutizzazioni in tale stadio) per cui si è assunto che i pazienti in tale stadio subiscano solo i costi diretti non legati a riacutizzazioni e limitatamente a visite, esami e 


\begin{tabular}{|c|c|c|c|c|c|c|}
\hline & & & Stadio I & Stadio II & Stadio III & Stadio IV \\
\hline & \multicolumn{6}{|l|}{ Costi Diretti } \\
\hline & \multirow[t]{3}{*}{ Ricoveri } & non associato a riacutizzazione & - & - & - & - \\
\hline & & associato a riacutizzazione & - & $1.346,20$ & $1.560,40$ & $2.828,80$ \\
\hline & & totale & - & $1.346,20$ & $1.560,40$ & $2.828,80$ \\
\hline & \multirow[t]{3}{*}{ Visite } & non associato a riacutizzazione & 147,67 & 147,67 & 162,53 & 164,55 \\
\hline & & associato a riacutizzazione & - & 43,13 & 47,47 & 48,05 \\
\hline & & totale & 147,67 & 190,80 & 210,00 & 212,60 \\
\hline & \multirow[t]{3}{*}{ Esami } & non associato a riacutizzazione & 80,61 & 80,61 & 82,15 & 93,05 \\
\hline & & associato a riacutizzazione & - & 44,79 & 45,64 & 51,70 \\
\hline & & totale & 80,61 & 125,40 & 127,79 & 144,74 \\
\hline & \multirow{3}{*}{$\begin{array}{l}\text { Farmaci } \\
\text { antibiotici }\end{array}$} & non associato a riacutizzazione & - & - & - & - \\
\hline & & associato a riacutizzazione & - & 174,89 & 200,26 & 217,38 \\
\hline & & totale & - & 174,89 & 200,26 & 217,38 \\
\hline & \multirow[t]{3}{*}{ Teofillina } & non associato a riacutizzazione & 54,56 & 54,56 & 54,56 & 54,56 \\
\hline & & associato a riacutizzazione & - & - & - & - \\
\hline & & totale & 54,56 & 54,56 & 54,56 & 54,56 \\
\hline & \multirow{3}{*}{$\begin{array}{l}\text { Beta2- } \\
\text { agonisti a } \\
\text { b.d.a }\end{array}$} & non associato a riacutizzazione & 82,70 & 82,70 & 100,98 & 113,32 \\
\hline & & non associato a riacutizzazione & - & 21,45 & 26,19 & 29,39 \\
\hline & & totale & 82,70 & 104,15 & 127,17 & 142,71 \\
\hline & \multirow[t]{3}{*}{ Altri farmaci } & non associato a riacutizzazione & 161,94 & 161,94 & 185,43 & 201,27 \\
\hline & & associato a riacutizzazione & - & 9,76 & 11,18 & 12,13 \\
\hline & & totale & 161,94 & 171,70 & 196,60 & 213,41 \\
\hline & \multirow{3}{*}{$\begin{array}{l}\text { Ossigeno- } \\
\text { terapia }\end{array}$} & non associato a riacutizzazione & - & 354,73 & 853,99 & $2.561,45$ \\
\hline & & associato a riacutizzazione & - & 60,07 & 144,61 & 433,75 \\
\hline & & totale & - & 414,80 & 998,60 & $2.995,20$ \\
\hline & \multirow[t]{3}{*}{ Ventilazione } & non associato a riacutizzazione & - & 24,50 & 89,59 & 340,54 \\
\hline & & associato a riacutizzazione & - & 4,10 & 15,01 & 57,06 \\
\hline & & totale & - & 28,60 & 104,60 & 397,60 \\
\hline & \multirow{3}{*}{$\begin{array}{l}\text { Terapie } \\
\text { riabilitative }\end{array}$} & non associato a riacutizzazione & - & 11,67 & 63,36 & 57,43 \\
\hline & & associato a riacutizzazione & - & 2,85 & 15,46 & 14,02 \\
\hline & & totale & - & 14,52 & 78,83 & 71,45 \\
\hline & \multirow[t]{4}{*}{ TOTALE } & non associato a riacutizzazione & 527,48 & 918,38 & $1.592,59$ & $3.586,18$ \\
\hline & & associato a riacutizzazione & - & $1.707,24$ & $2.066,22$ & $3.692,27$ \\
\hline & & totale & 527,48 & $2.625,62$ & $3.658,81$ & $7.278,44$ \\
\hline & & per riacutizzazione & - & $1.219,46$ & $1.475,87$ & $2.637,33$ \\
\hline & \multicolumn{6}{|c|}{ Costi Indiretti } \\
\hline & \multirow{3}{*}{$\begin{array}{l}\text { Assenze } \\
\text { dal lavoro }\end{array}$} & non associato a riacutizzazione & - & 2,63 & 3,66 & 7,28 \\
\hline & & associato a riacutizzazione & - & 6,57 & 9,15 & 18,21 \\
\hline & & totale & - & 9,20 & 12,82 & 25,49 \\
\hline & \multirow{3}{*}{$\begin{array}{l}\text { Assenze } \\
\text { dei familiari }\end{array}$} & non associato a riacutizzazione & - & 13,14 & 18,31 & 36,42 \\
\hline & & associato a riacutizzazione & - & 32,37 & 45,11 & 89,73 \\
\hline & & totale & - & 45,51 & 63,41 & 126,15 \\
\hline & \multirow[t]{4}{*}{ TOTALE } & non associato a riacutizzazione & - & 15,77 & 21,97 & 43,71 \\
\hline & & associato a riacutizzazione & - & 38,94 & 54,26 & 107,94 \\
\hline & & totale & - & 54,70 & 76,23 & 151,64 \\
\hline & & per riacutizzazione & - & 27,81 & 38,76 & 77,10 \\
\hline & \multicolumn{6}{|c|}{ Totale costi diretti e indiretti } \\
\hline \multirow{4}{*}{$\begin{array}{l}\text { Prospetto delle singole } \\
\text { voci di costo, suddivise } \\
\text { per gravità della } \\
\text { patologia e tipologia di } \\
\text { risorsa consumata } \\
\text { (costi in euro) }\end{array}$} & & non associato a riacutizzazione & 527,48 & 934,15 & $1.614,56$ & $3.629,88$ \\
\hline & & associato a riacutizzazione & - & $1.746,18$ & $2.120,48$ & $3.800,21$ \\
\hline & & totale & 527,48 & $2.680,33$ & $3.735,04$ & $7.430,09$ \\
\hline & & per riacutizzazione & - & $1.247,27$ & $1.514,63$ & $2.714,43$ \\
\hline
\end{tabular}




\begin{tabular}{|c|c|c|c|c|c|}
\hline Farmaco & Confezione & $\begin{array}{l}\text { Costo } \\
\text { confezione }\end{array}$ & $\begin{array}{c}\text { Posologia } \\
\text { trial }\end{array}$ & $\begin{array}{l}\text { Durata per } \\
\text { confezione }\end{array}$ & $\begin{array}{c}\text { Costo annuo } \\
\text { trattamento }\end{array}$ \\
\hline SF & GSK Seretide $50 / 500 \mu \mathrm{g} 60 \mathrm{~d}$ & 81,43 & $\begin{array}{c}50 / 500 \mu g \\
2 \text { volte/giorno }\end{array}$ & 1 mese & 977,16 \\
\hline FB & $\begin{array}{c}\text { Astrazeneca Symbicort } \\
160 / 4,5 \mu \mathrm{g} 120 \mathrm{~d}\end{array}$ & 63,07 & $\begin{array}{c}160 / 4,5 \mu \mathrm{g} \\
2 \text { inalazioni } \\
2 \text { volte/giorno }\end{array}$ & 1 mese & 756,84 \\
\hline $\mathrm{F}$ & GSK Flixotide $500 \mu \mathrm{g} 60 \mathrm{~d}$ & 57,78 & $\begin{array}{c}500 \mu \mathrm{g} \\
2 \text { volte/giorno }\end{array}$ & 1 mese & 693,36 \\
\hline S & GSK Serevent $50 \mu \mathrm{g} 60 \mathrm{~d}$ & 37.05 & $\begin{array}{c}50 \mu \mathrm{g} \\
2 \text { volte/giorno }\end{array}$ & 1 mese & 444,60 \\
\hline
\end{tabular}

farmaci. Per tali valori si sono utilizzati quelli dello stadio II.

Per calcolare la formazione dei costi nel tempo, all'interno del modello di Markov viene applicato un costo legato alla permanenza di un individuo in uno stato, secondo diverse prospettive:

- prospettiva del paziente $\rightarrow$ solo i costi indiretti;

- prospettiva del SSN $\rightarrow$ solo i costi diretti;

- prospettiva della società $\rightarrow$ costi diretti e indiretti.
In ogni stato del modello di Markov, su ciascun ramo dell'albero decisionale, il costo viene calcolato come:

costo non legato a riacutizzazione + costo per riacutizzazione * numero di riacutizz. pop. iniziale $* R R$ riacutizzazioni + costo farmaceutico del trattamento attivo - costo farmaci $\beta 2$-agonisti a b.d. * RR uso di "relief medications"

I costi farmaceutici dei trattamenti attivi considerati (dal sito www.ministerosalute.it consultato il 8/6/05) sono riportati in Tabella VII.

\section{Tabella VII}

Costi farmaceutici dei trattamenti attivi (costi in euro)

\begin{tabular}{|c|c|}
\hline Dato & Variazione \\
\hline FEV1 medio (\% pred.) della popolazione iniziale & $\pm 10 \%$ \\
\hline N medio riacutizzazioni/paziente/anno & $+100 \%-50 \%$ \\
\hline Declino capacità polmonare & $+/-50 \%$ \\
\hline Sopravivenza a 4 anni & $\mathrm{Cl}$ \\
\hline Riduzione riacutizzazioni & $\mathrm{Cl}$ rielab. \\
\hline GG liberi da sintomi & $+/-20 \%$ \\
\hline RR uso di relief medications & $+/-20 \%$ \\
\hline Costi diretti: ricoveri & $+/-20 \%$ \\
\hline Costi diretti: visite & $+/-20 \%$ \\
\hline Costi diretti: esami & $+/-20 \%$ \\
\hline Costi diretti: farmaci & $+/-20 \%$ \\
\hline Costi diretti: ossigenoterapia & $+/-20 \%$ \\
\hline Costi diretti: ventilazione & $+/-20 \%$ \\
\hline Costi diretti: terapie riabilitative & $+/-20 \%$ \\
\hline Costo farmaco & $+/-20 \%$ \\
\hline
\end{tabular}

\section{Tabella VIII}

Variabili utilizzate per analisi di sensibilità ad una via 


\section{Tabella IX}

Risultati principali del modello con l'orizzonte temporale

corrispondente all'intera durata della

simulazione: valori medi per paziente (costi in euro)

\begin{tabular}{cccccc}
\hline Opzione & Riacutizzazioni & $\begin{array}{c}\text { GG liberi } \\
\text { da sintomi }\end{array}$ & $\begin{array}{c}\text { Costo per } \\
\text { il SSN }\end{array}$ & $\begin{array}{c}\text { Costo per } \\
\text { il paziente }\end{array}$ & $\begin{array}{c}\text { Costo per } \\
\text { la società }\end{array}$ \\
\hline Controllo & 12,04 & 0 & $34.632,09$ & 715,74 & $35.347,83$ \\
\hline Salmeterolo & 10,07 & 55 & $33.369,28$ & 619,87 & $33.989,15$ \\
\hline Fluticasone & 10,14 & 37 & $34.754,38$ & 623,55 & $35.377,93$ \\
$\begin{array}{c}\text { Salmeterolo- } \\
\text { Fluticasone }\end{array}$ & 9,09 & 257 & $34.037,71$ & 256,89 & $34.037,71$ \\
$\begin{array}{c}\text { Formoterolo- } \\
\text { Budesonide }\end{array}$ & 9,66 & 220 & $33.944,51$ & 599,73 & $34.544,25$ \\
\hline
\end{tabular}

Si assume che la transizione tra due stati avvenga mediamente a metà ciclo. Pertanto nel passaggio da uno stato all'altro viene applicato un costo medio tra i due caratteristici dei due stati tra cui avviene la transizione. Nel caso di morte viene applicata la metà del costo dello stato di provenienza.

\section{Efficacia complessiva, costi totali, costo/efficacia e analisi di sensibilità}

La simulazione del modello, dunque, fornisce i costi totali e l'efficacia complessiva associati a ciascun ramo dell'albero decisionale. Di seguito vengono calcolati rapporti di costo efficacia incrementale per attribuire un costo ad ogni evento clinico evitato o unità di efficacia guadagnata rispetto alla strategia alternativa.

La robustezza dei risultati viene testata mediante una serie di analisi di sensibilità a una via (Tabella VIII); sui parametri la cui variazione è in grado di cambiare il risultato principale del confronto, viene inoltre condotta un' analisi del valore soglia.

Note:

- la variazione di $\pm 10 \%$ è stata applicata simultaneamente ai dati del FEV1 medio per ogni stadio GOLD della popolazione iniziale. La variazione è stata limitata al $10 \%$ perché una variazione maggiore avrebbe comportato uno spostamento di stadio;

- la variazione di $+100 \%$ e $-50 \%$ è stata applicata simultaneamente ai numeri medi di riacutizzazioni/paziente/anno per ogni stadio GOLD della popolazione iniziale. La variazione $\mathrm{di}+100 \%$ porta il valore medio di base di 0,7 riacutizzazioni/paziente/anno al valore 1,4 che è stato sperimentato dal gruppo dei pazienti dello studio ICE;

\section{Tabella $X$}

Costo efficacia incrementale delle associazioni precostituite vs. salmeterolo (costi in euro)

* Euro/riacutizzazione evitata

**Euro/gg libero da sintomi guadagnato
}

\begin{tabular}{cccc} 
& Riacutizzazioni & GG liberi da sint & Costo diretto tot \\
\hline $\begin{array}{c}\text { Salmeterolo- } \\
\text { Fluticasone } \\
\text { Salmeterolo }\end{array}$ & 9,09 & 257 & $34.037,71$ \\
Incrementale & 10,07 & 55,05 & $33.369,28$ \\
ICER & 0,98 & 202 & 668,43 \\
& $679,55^{*}$ & $3,31^{* *}$ & \\
Formoterolo- & & & $33.944,51$ \\
Budesonide & 9,66 & 220 & $33.369,28$ \\
Salmeterolo & 10,07 & 55,05 & 575,23 \\
Incrementale & 0,41 & 165 & \\
ICER & $1.392,38^{*}$ & $3,48^{* *}$ & \\
\hline
\end{tabular}


- la variazione di -50\% del declino della capacità polmonare $(45 \mathrm{ml} / \mathrm{anno})$ porta al valore medio (30 ml/anno) per un non fumatore;

- l'intervallo di variazione della riduzione delle riacutizzazioni dovute al trattamento attivo è stato ricavato adattando l'intervallo di confidenza pubblicato sullo studio Calverley per $\mathrm{RR}=0,746$ (CI 0,643-0,865) al valore utilizzato $R R=0,7$ (CI 0,603-0,812).

\section{RISULTATI}

\section{Scenario base}

La sopravvivenza media del campione dall'inizio della simulazione risulta essere di circa 11,5 anni in tutti i rami del modello.

La Tabella IX riporta i risultati principali del modello, espressi come valori medi per paziente, con l'orizzonte temporale corrispondente all'intera durata della simulazione.

Per la scarsa significatività della stima dei costi indiretti, pressoché equivalenti nei vari rami, con eccezione di SF, che è associato a costi per il paziente pari a meno della metà delle alternative, tutte le analisi successive saranno riferite ai soli costi diretti, a carico del SSN.

Nella prospettiva del SSN, tutti i trattamenti attivi, con la sola eccezione del fluticasone, si rivelano dominanti dal punto di vista farmacoeconomico, cioè producono migliori risultati clinici a un costo inferiore del controllo.

La performance farmacoeconomica delle due associazioni fisse di corticosteroidi e broncodilatatori risulta pressoché sovrapponibile, con una lieve superiorità clinica di salmeterolofluticasone.

Il trattamento con salmeterolo risulta essere associato a costi leggermente inferiori rispetto alle associazioni precostituite, ma anche a risultati clinici inferiori. La Tabella $X$ riporta il calcolo della costo efficacia incrementale di SF e FB rispetto a $S$, che quantifica l'investimento necessario ad ottenere un'unità di beneficio clinico aggiuntiva (riacutizzazione evitata o giorno libero da sintomi guadagnato).

\section{Analisi di sensibilità}

Il risultato principale fornito dal modello è la dominanza farmacoeconomica delle associazioni fisse di corticosteroide e broncodilatatore inalatori rispetto al controllo. Nelle Figure 3 e 4 viene rappresentata la sensibilità delle due dimensioni principali di questo risultato - minor numero di riacutizzazioni e minor costo - alle variazioni dei cinque parametri principali per ampiezza della variazione di risultato indotta. Come evidenziato nei grafici, la strategia SF perde la dominanza, ossia risulta maggiormente efficace ma anche più costosa rispetto al ramo di controllo, in seguito alla variazione di 4 variabili:

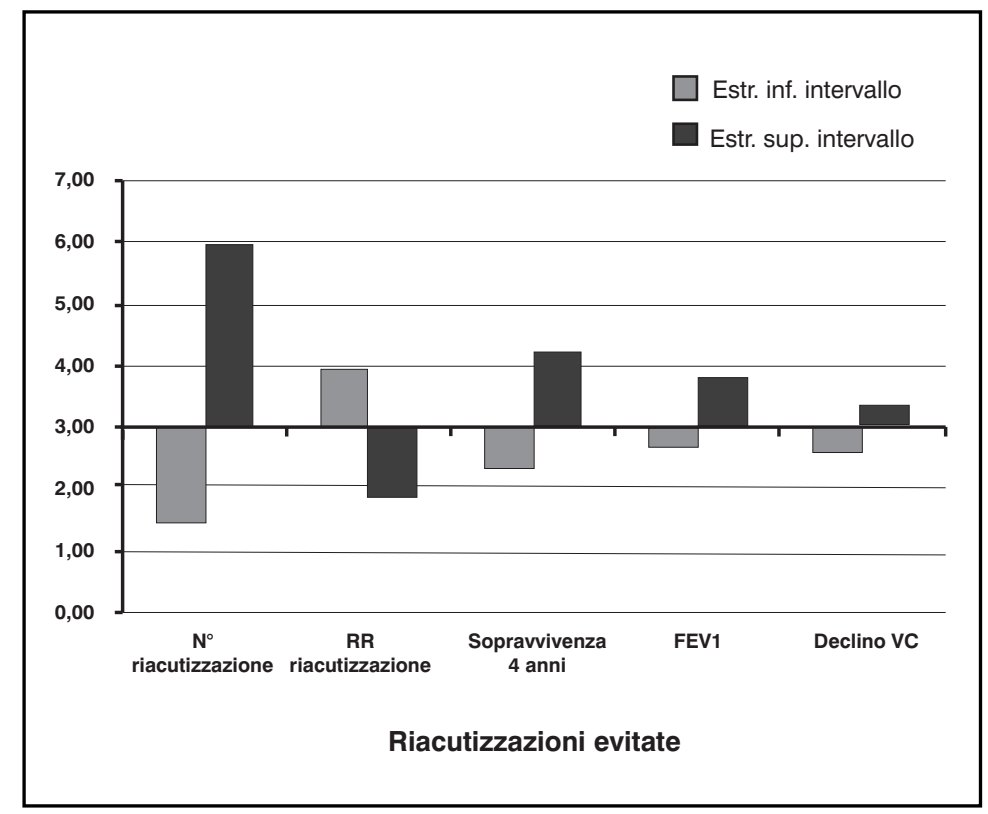

Figura 3

Prime cinque variabili per ampiezza della variazione prodotta sul risultato relativo a riacutizzazioni/paziente evitate (confronto SF vs C; valore base 2,95 riacutizzazioni/paziente)

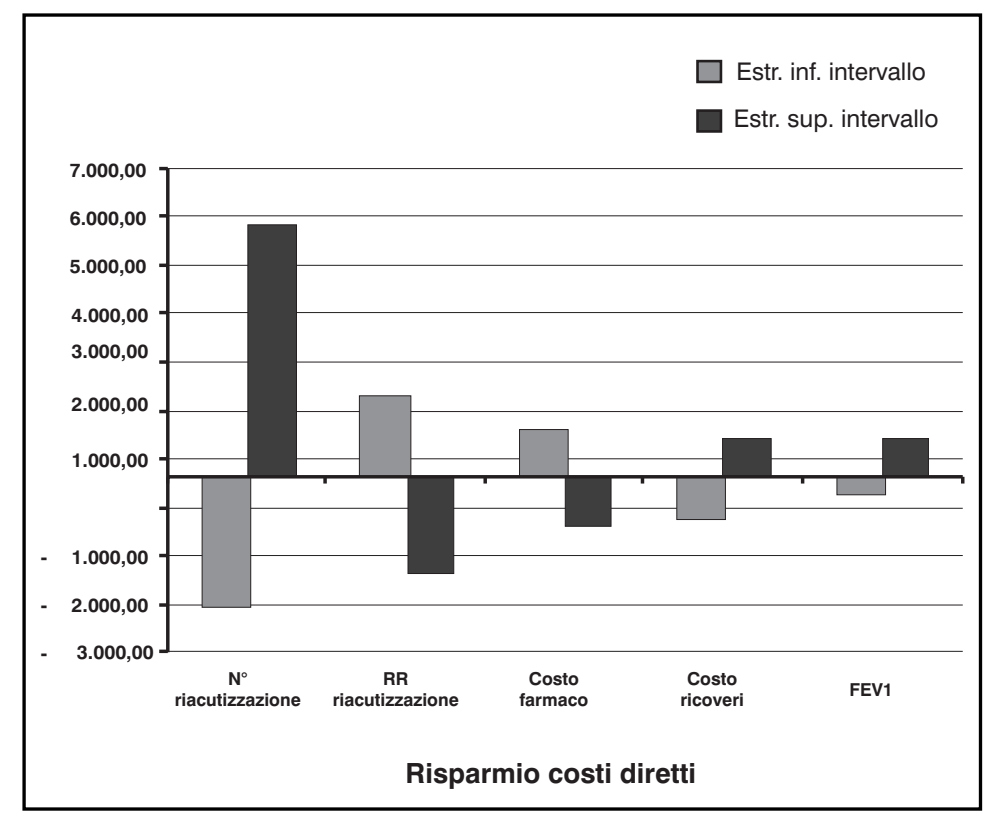

Figura 4

Prime cinque variabili per ampiezza della variazione prodotta sul risultato relativo al risparmio di costi diretti (confronto SF vs C; valore base 594,38 euro)

- riduzione del numero medio riacutizzazioni/paziente/anno;

- aumento del RR di riacutizzazione con SF;

- aumento del costo del farmaco;

- riduzione dei costi di ricovero.

Per questi quattro casi è riportato il calcolo della costo efficacia agli estremi dell'intervallo di variabilità testato, che danno un'indicazione dei "best case" e "worst case". 


\begin{tabular}{lccccc}
\multicolumn{1}{c}{ Dato } & Valore base & C/E base & $\begin{array}{c}\text { Valore } \\
\text { best case }\end{array}$ & $\begin{array}{c}\text { C/E } \\
\text { best case }\end{array}$ & $\begin{array}{c}\text { Valore } \\
\text { worst case } \\
\text { worst case }\end{array}$ \\
\hline $\begin{array}{l}\text { N medio } \\
\text { riacutizzazioni } \\
\text { /paziente/anno }\end{array}$ & 0,67 & SF dominante & 1,33 & SF dominante & 0,33 \\
RR riacutizzazioni & 0,700 & SF dominante & 0,603 & SF dominante & 0,812 \\
Costi diretti: ricoveri & $1.862,00$ & SF dominante & $2.234,40$ & SF dominante & $1.489,60$ \\
\hline
\end{tabular}

Tabella XI

Costo efficacia del "worst case" e "best case" (costi in euro)

È stata, inoltre, sviluppata un'analisi di soglia, ossia il calcolo del valore che annulla il vantaggio economico associato a $\mathrm{SF}$.

Per esemplificare il significato di questi valori, prendiamo il caso del RR di riacutizzazione: il valore di soglia di 0,734 indica che $\mathrm{SF}$, oltre ad essere clinicamente superiore al controllo, è anche associato a minori costi totali fintanto che è in grado di prevenire il $26,6 \%$ $(1,0-0,734=0,266)$ delle riacutizzazioni; a livelli di efficacia minore, $i$ benefici clinici aggiuntivi devono essere scambiati con un aumento di costi, pari a 733,33 euro/riacutizzazione evitata in corrispondenza del caso peggiore, nello specifico corrispondente all'estremo superiore dell'intervallo di confidenza del RR di riacutizzare con SF ottenuto nello studio di Calverley et al.

\section{CONCLUSIONI}

L'obiettivo principale di questo lavoro era la valutazione delle conseguenze cliniche ed economiche delle raccomandazioni GOLD in materia di trattamento dei pazienti con BPCO grave e molto grave nel contesto sanitario italiano. Mentre non vi sono dubbi sulla superiorità clinica dell' approccio consigliato, che si basa sulle migliori evidenze scientifiche, in accordo con i principi dell'EBM (EvidenceBased Medicine), i riflessi della strategia GOLD sulla spesa sanitaria sostenuta dal SSN per la gestione dei pazienti con BPCO in stadio III e IV non erano noti. Al fine di contribuire a chiarire questo aspetto, abbiamo costruito un modello farmacoeconomico utilizzando parametri clinici ottenuti da trial internazionali e variabili socio-demografiche ed economiche relative al nostro Paese.

I risultati del modello indicano che il trattamento degli stadi GOLD III e IV con associazioni precostituite di $\beta$-agonisti e corticosteroidi per via inalatoria è più conveniente delle strategie terapeutiche alternative, in quanto i superiori costi farmaceutici sono compensati, del tutto o in massima parte a seconda delle assunzioni postulate, dalla riduzione dei costi associati ai ricoveri ospedalieri.

In altri termini, secondo il nostro modello, nella terapia della BPCO le priorità cliniche coincidono con quelle economiche, per cui con l'utilizzo delle associazioni di broncodilatori e corticosteroidi è possibile assicurare una miglior qualità di vita ai pazienti broncopneumopatici gravi senza indurre un aumento della spesa sanitaria, se non addirittura con un concomitante risparmio.

\begin{tabular}{lcc}
\hline \multicolumn{1}{c}{ Dato } & Valore base & Valore soglia \\
\hline N medio riacutizzazioni/paziente/anno & 0,67 & 0,59 \\
RR riacutizzazioni & 0,700 & 0,734 \\
Costi diretti: ricoveri & $1,862,00$ & $1.584,00$ \\
Costo farmaco & 81,43 & 91,28 \\
\hline
\end{tabular}




\section{BIBLIOGRAFIA}

1. De Marco R., Accordini S., Cerveri I., Corsico A., Sunyer J., Neukirch F., Kunzli N., Leynaert B., Janson C., Gislason T., Vermeire P., Svanes C., Anto M.J., Burney P. An international Survey of chronic obstructive pulmonary disease in young adults according to GOLD stages. Thorax 2004; 59: 120-125.

2. Viegi G., Scognamiglio A., Baldacci S., Pistelli F., Carrozzi L. Epidemiology of Chronic Obstructive Pulmonary Disease. Respiration 2001; 68: 4-19.

3. ISTAT. Indagine Multiscopo sulle famiglie: condizioni di salute e ricorso ai servizi sanitari anno 1999-2000.

4. Price D., Tinkelman D.G., Nordyke R.J., Isonaka S., Halbert R.J., Severity distribution of copd in primary care. 2004, AbstractERS4L1_2562.

5. Antonelli-Incalzi R., Imperiale C., Bellia V., Catalano F., Scichilone N., Pistelli R., Rengo F. Do GOLD stages of COPD severità really correspond to differences in health status? Eur Respir J 2003; 22: 444-449.

6. Burge S., Wedzicha J.A. COPD exacerbations: definitions and classifications. Eur Respir J 2003; 21: Suppl. 41, 46s$53 \mathrm{~s}$.

7. Fletcher C.M., Peto R., Tinker C.M., Speizer F.E. The natural history of chronic bronchitis and emphysema. Oxford: Oxford University Press, 1976.

8. ISTAT. Annuario statistico italiano 2003.

9. Domingo-Salvany A., Lamarca R., Ferrer M., Garcia-Aymerich J., Alonso J., Felez M., Khalaf A., Marrades R.M., Monsò E., Serra-Batlles J., Antò J.M. Health-related Quality of Life and Mortality in Male Patients with Chronic Obstructive Pulmonary Disease. Am J Respir Crit Care Med 2002; 166: 680-685.

10. Crapo RO, Morris AH, and Gardner RM. Reference Spirometric Values Using Techniques and Equpment That Meet ATS Recommendations. Am. Rev. of Respir. Dis. 1981; 123:659-664.

11. Crapo RO and Morris AH. Standardized Single-Breath Normal Values for Carbon Monoxide Diffusing Capacity. Am. Rev. of Respir. Dis. 1981; 123:185-189.

12. Crapo RO, Morris AH, Clayton PD, and Nixon CR. Lung Volumes in Healthy Nonsmoking Adults. Bull. Europ. Physiopathol. Respir. 1982; 18:419-425.

13. Calverley P., Pauwels R., Vestbo J., Jones P., Pride N., Gulsvik A., Anderson J., Maden C. Combined salmeterol and fluticasone in the treatment of chronic obstructive pulmonary disease: a randomised controlled trial. The Lancet 2003; 361:449-456

14. Szafranki W., Cukier A., Ramirez A., Menga G., Sansores R., Nahabedian S., Peterson S., Olsson H. Efficacy and safety of budesonide/formoterol in the management of chronic ostructive pulmonary disease. Eur Respir J 2003; 21: 74-81

15. Dal Negro R.W., Pomari C., Tognella S., Micheletto C. Salmeterol \& Fluticasone $50 \mu \mathrm{g} / 250 \mu \mathrm{g}$ bid in combination provides a better long-term control than salmeterol $50 \mu \mathrm{g}$ bid alone and placebo in COPD patients already treated with theophylline. Pulmonary Pharmacology \& Therapeutics. 2003; 16: 241-246

16. Calverley P.M., Boonsawat W., Cseke Z., Zhong N., Peterson S., Olsson H. Maintenance therapy with budesonide and formoterol in chronic obstructive pulmonary disease. Eur Respir J 2003; 22: 912-919.

17. Lucioni C., Donner C.F., De Benedetto F., Lusuardi M., Mazzi S., Paggiaro P.L., Sanguinetti C.M. I costi della broncopneumopatia cronica ostruttiva: la fase prospettica dello studio ICE (Italian Costs for Exacerbations in COPD) - In Press

18. Dal Negro R.W., Rossi A., Cerveri I. The burden of COPD in Italy: results from the Confronting COPD survey. Resp Med 2003; 97 (Suppl. C): S43-S50 